\title{
STAY-HEALTHY: AN EXPERT SYSTEM TO SUGGEST A HEALTHY DIET
}

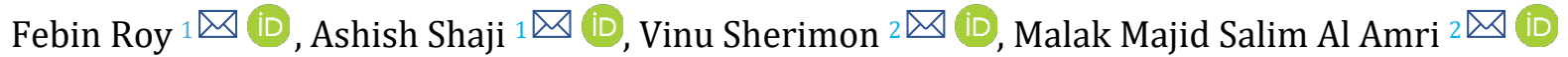 \\ ${ }^{1}$ Department of Computer Science and Engineering, Saintgits College of Engineering, Kottayam, Kerala, India. \\ ${ }^{2}$ Department of Information Technology, University of Technology and Applied Sciences, Muscat, Sultanate \\ of Oman.
}

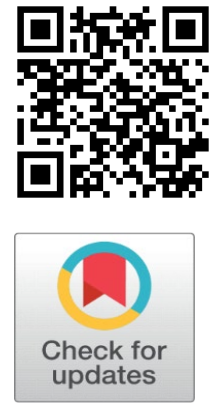

Received 10 November 2021

Accepted 15 December 2021

Published 20 January 2022

\section{CorrespondingAuthor}

Vinu Sherimon,

vinusheri@hct.edu.om

DOI 10.29121/IJOEST.v6. i1.2022.262

Funding: The research leading to these results has received funding from the Research Council (TRC) of the Sultanate of Oman under the Block Funding Program BFP/URG/ICT/20/031.

Copyright: (C) 2022 The Author(s). This is an open access article distributed under the terms of the Creative Commons Attribution License, which permits unrestricted use, distribution, and reproduction in any medium, provided the original author and source are credited.

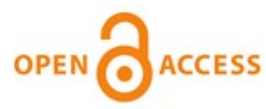

\section{ABSTRACT}

In this time of sudden outbreaks of illnesses and new viruses, people try to seek out more healthy and better lives to protect their fitness in all viable ways. As ways as an amateur character care, he/she isn't aware of the shape of ingredients and therefore the big variety of energy to eat which could lead on him/her to a healthful life, especially people that are suffering from persistent non-Communicable illnesses (NCD) which include cardiovascular illnesses, hypertension, diabetes etc. This study proposes the development of a knowledgeable gadget that shows a customized everyday weight loss program plan, specifically for citizens suffering from NCD. As a part of this study, we've developed a recommendation system considering the above facts. Recommendation systems are considered an efficient technology that helps users to regulate their healthy diet and be free from the NCDs.

Keywords: Non-Communicable Diseases (NCD), Recommendation Systems, Expert System, Healthy Diet, Customized Health Plan.

\section{INTRODUCTION}

As we all know foods are essential for human to sustain their life. Foods are directly related to one's body and socio-mental health. However, many people today are affected by many diseases due to unhealthy diets. As per the studies conducted by World Health Organization (WHO) GBD (2015) shows that NCD kills 41 million people in a year, around 71\% of total deaths. Noncommunicable diseases include mainly heart attacks, hypertension, diabetics, obesity, cancer etc. Unhealthy healthy diets and lack of proper exercise leads to High blood pressure, elevated blood sugar, high blood fat content, and obesity. Furthermore, many of us, whether adults or children, like eating unhealthy junk food. These foods appeal to everyone because of the flavor enhancers applied to them, which have a negative impact on our bodies. Every person on the planet desires to live a good life. Everyone can stay healthy if they eat a well-balanced diet and exercise regularly. Every single one of us isn't a nutritionist. It is extremely difficult for the average person to comprehend and consume the proper calorie quantities required by our bodies.

The main focus of our research paper is to propose a dietary based recommendary system for the common people so as they can have a healthier diet as well as they will be from the threatening of NCD's. The proposed recommendary system, Stay-Health will consider the health characteristics of the users to achieve the personalized food recommendation. 
The rest of the paper is organized as follows: Related work is presented in Section 2. Section 3 describes the methodology of the research which includes dataset details, techniques used for building a Recommendation System, architecture of the proposed application, and K-means clustering algorithm. Experimental results are given in Section 4 and Conclusion is given in Section 5. Section 6 includes Future work followed by References.

\section{RELATED WORK}

Many pieces of work have already been done in the field of recommendation systems using various algorithms and different methodologies by different researchers.

Phanich et al. (2010) have developed a diet recommendary system for diabetic patients by using the technique of nutrition therapy. Typically, diabetic patients are required to avoid fat and sugar from diabetics. The paper developed a set of guidelines recommending diet therapy as the main treatment for diabetic patients with various dietary restrictions. The author uses cluster analysis and selforganization techniques to develop FRS for diabetic patients to provide optimal food intake recommendations.

Research given in Seo et al. (2020) suggested that people with gestational diabetes (GDM use nutrition apps on their phones to improve their health. The app records the amount of food consumed, checks the data, and creates diet reports. Nutritionist training on food selection, meal planning, meal billing, etc. In addition, personalized messages are sent twice a month, and training materials are sent once a week. Regarding the nutritional content of various foods, the elements necessary for consumption during pregnancy, and some related safety measures, similar courses are also offered after delivery. The study's finding is that these smartphone apps are particularly useful for women who have been identified with GDM.

The research presented in Coumans et al. (2020) involves the development of the nutrition of the elderly and the development of the personal nutrition system. The participants in the study were 63 senior persons in good health and 92 senior citizens who had difficulty swallowing food. Survey questionnaires were utilized to gather data, and the researchers were able to pinpoint the factors that contributed to lower calorie intake and anorexia. Then they proposed a healthy and personalized diet. Studies have shown that physical activity, the weight of the elderly, and high protein requirements are some of the most important factors to consider when developing a customized recipe. Developed "MiranaBot", which can identify the eating habits, food consumption, etc. of the elderly, and recommend healthy eating.

This paper Manoharan and Sathish (2020) introduces the recommendation system of Kclique's built-in deep learning classifier to provide nutrition advice to patients. Kclique is included in the recommender to improve the accuracy of and the accuracy of deep learning classifiers (closed repeating units). The data set for empirical analysis of the developed system is a patient data set collected on the Internet. About 50 patients' 13 characteristics of various diseases and 8 feature sets were collected in hospitals. Product information. Before being applied to deep learning classifiers, all these features are encoded and grouped into multiple clusters and compares the best observation accuracy and accuracy of the experimentally developed system with machine learning methods (such as logistic regression and naive Bayes analysis) and other deep learning classifiers (such as MLP and RNN) to Determine the performance learning based on Kclique's deep learning-recommended display classifier. 
The researchers Li and Ko (2007) came up with a diet recommendary system for diabetic patients. They suggest the creation of a diabetic diet ontology that is automated. Our approaches include hierarchical clustering algorithms (HCA) for building an ontology skeleton, intersection naming for class naming, and granular ranking and placement for instance ranking. Nutritionists will benefit from our method since it will allow them to create new food categories and rank diet recommendations in a hierarchical order.

\section{METHODOLOGY}

\subsection{DATASET}

We have used an open dataset for this project as input for the machine learning algorithm. The dataset consists of various commonly used food items with their nutrition values including fats, calories, iron, vitamins etc. For improved diet recommendations much, large dataset can be used.

\subsection{TECHNIQUES USED FOR BUILDING A RECOMMENDATION SYSTEM}

\subsubsection{CONTENT-BASED FILTERING METHOD}

Content-based filtering may be a domain-specific algorithmic rule that focuses additional on evaluating the attributes of things to form predictions. once providing files cherish pages, posts, and messages, content-based filtering methods are possible be content-based filtering methods. it's suggested that a person's personal information be supported by the characteristics of the person's antecedently studied info.

\subsubsection{COLLABORATIVE BASED FILTERING METHOD}

Collaborative filtering may be a domain-independent content prediction methodology that can't be delineated simply and adequately exploitation data like movies and music. The rule of collaborative filtering is to form info (custom project array) that contains the user settings of the project. cooperative filtering is a method of mechanically predicting (filtering) user interests by assembling information regarding preferences or the preferences (collaboration) of the many users. the most assumption of the collaborative filtering method is that if person $A$ and person $B$ have a constant opinion, then topic A is a lot of probably to carry B' opinion on a special topic than a haphazardly selected person.

\subsubsection{HYBRID BASED FILTERING METHOD}

Several packages integrate memory-primarily based totally and modelprimarily totally based CF algorithms; they triumph over the constraints of proprietary CF techniques and enhance prediction performance. Usually, maximum business advice engines are mixed, including Google's information advice engine.

\subsection{ARCHITECTURE}

Our proposed StayHealthy application has been made up with the technique of machine learning. 


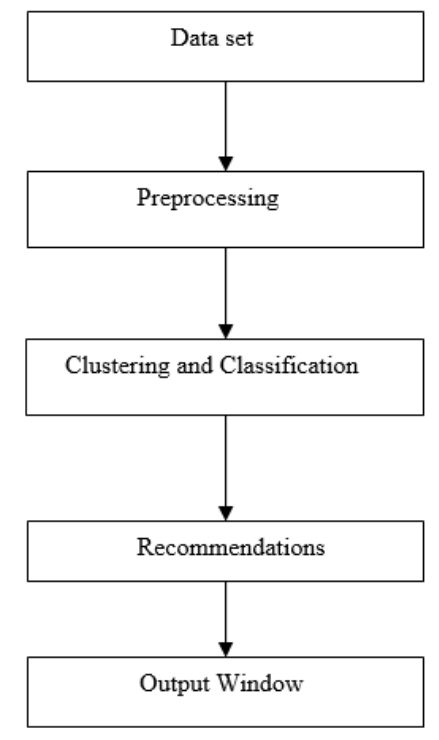

Figure 1 Architecture of Stay-Health

Dataset is being preprocessed first and we use certain rules to find the patient have any diseases or not. By using this result the system will forward to the next step. Stay-Health recommendation system uses the unsupervised machine learning algorithm, $\mathrm{K}$ means clustering and further the output is being classified using the classifier. We are using a hybrid model of recommendation system.

\subsection{K MEANS CLUSTERING}

Clustering's major purpose is to partition a dataset or set of data into numerous disjoint datasets based on similarities in the data. Each subset is referred to as a "cluster," which means that items inside the same "cluster" have a higher level of similarity on certain qualities, while objects from completely distinct "clusters" have a lower level of similarity.

K Means is one of the popular clustering algorithms which have been developed by several independent observations by different researchers by the mid of $20^{\text {th }}$ century Pérez-Ortega (2019). This algorithm divides the data into $\mathrm{n}$ disjoint subsets as $(X=\{x 1, x 2, \ldots, x n\})$ into k clusters, such that $y \cap y^{\prime}=\emptyset$. The similarities of the various cluster are measured by SSE (sum of squared errors). The algorithm divides the data into clusters with the smallest feasible squared distances between them and the cluster centroid. Centroids are the mean of all data points in the given cluster. The algorithm works as follows:

- Specify the number of clusters, $\mathrm{k}$

- To begin, combine the dataset and then select $\mathrm{k}$ data points at random for the centroids without altering them.

- Iterate till the centroids do not change. That is the data point clustering does not vary.

- Place each data to the cluster that is closest to it (centroid).

Then we must iterate the different values of $\mathrm{k}$ and find the optimal cluster. Here in our model, the optimal cluster is 3 . 


\section{EXPERIMENTAL RESULTS}

A Diet recommendary application has been developed using machine learning algorithms and python graphical interface tkinter. As mentioned earlier we have been used the K means algorithm and random forest classifier for the classification purposes. Initially, the application receives input from the user which includes the height of the person, weight of the person, blood pressure, cholesterol level, diabetics level, age of the person. The system makes use of some rules to classify whether the patient is affected by one or more diseases, or he is healthy. K means clustering algorithm is being used for data clustering and the clustered data is then classified using a random forest classifier. The following figure shows the input section of the Stay-Health application.

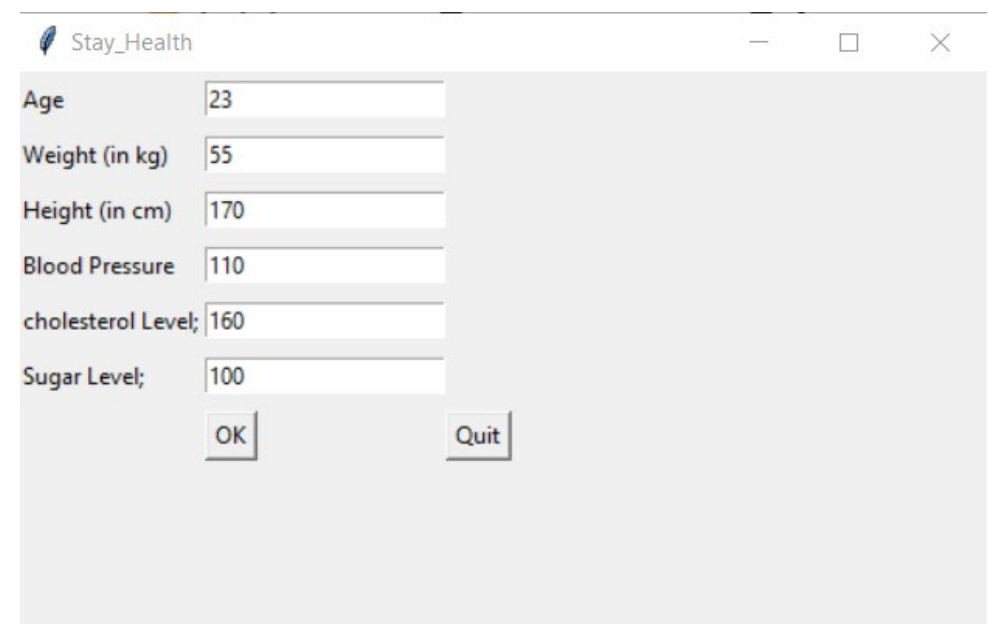

Figure 2 User input of Stay-Healthy

According to the rules and with the machine learning algorithms, the system recommends the food chart for the user (Figure 3). This system can be used as a diet recommendary system for NCD Patients with some modifications.

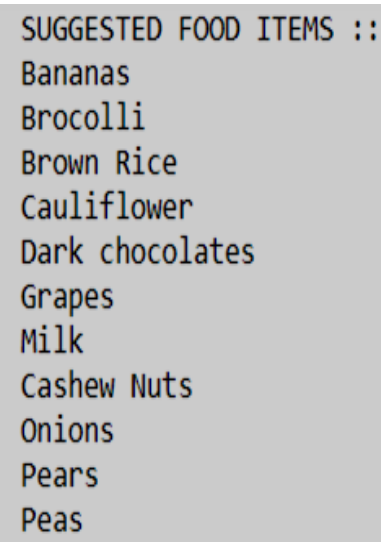

Figure 3 Recommended Diet Chart

\section{CONCLUSION}

People are striving a longer and sustainable life in this era of unforeseen disease outbreaks and new viruses to protect their health in any manner feasible. A novice individual, those diagnosed with chronic non-communicable diseases (NCDs) such as cardiovascular illnesses, hypertension, diabetes, and others, is unaware of the 
types of nutrients necessary for a healthy existence. This study proposed the construction of an expert system that recommends a personalized daily meal plan, particularly for those with NCD.

Existing research on nutrition consulting systems is essential to help users choose a diet that suits their interests and health conditions. These studies use information about user profiles and recipes to provide dietary advice. It is widely acknowledged that the suitability and reliability of user information and food nutritional value information have a significant role in the quality of suggestions. Also, our system is currently on progress with developing phase, so reliable output will be only based on the app which is the final output.

\section{FUTURE WORK}

With future improvements to the methods used in this project, a complete and more accurate ingredient vocabulary will be created. Although the application works well on the local computer, the next step is to make it available on the network and have improved features. For example: adding additional dietary advice to choose ingredients rich in anti-cancer molecules, while reducing negative interactions with drugs other than anti-cancer drugs. The application will soon be made available as a mobile app so that more people can access the application.

The user base should be very massive to provide a vulnerable comparison between the diet patterns among users, since the dataset only contains preset foods the addition of more users requires the nutritional values regarding their new food items. We can improve the recommendations by adding more food items to the dataset, so that a variety of diet recommendation can be given to the indented users.

\section{REFERENCES}

Coumans, J. M., Bolman, C. A., Friederichs, S. A., Oenema, A., \& Lechner, L. (2020). Development and Testing of a Personalized Web-Based Diet and Physical Activity Intervention Based on Motivational Interviewing and the SelfDetermination Theory : Protocol for the MyLifestyleCoach Randomized Controlled Trial. JMIR Research Protocols, 9(2), e14491. Retrieved from https://doi.org/10.2196/14491

Dr Samuel Manoharan, Prof Sathish (2020). Diet Recommendation System using K Clique and Deep learning classifiers, Journal of Artificial Intelligence and Capsule Networks, Vol.02/ No. 02 Pages : 121-130. Retrieved from https://doi.org/10.36548/jaicn.2020.2.005

GBD (2015) Risk Factors Collaborators. Global, regional, and national comparative risk assessment of 79 behavioural, environmental and occupational, and metabolic risks or clusters of risks, 1990-2015 : a systematic analysis for the Global Burden of Disease Study 2015. Lancet, 2016 ; 388(10053) :16591724. Retrieved from https://doi.org/10.1016/S0140-6736(16)31679-8

Huan-Chung Li; Wei-Min Ko (2007). Automated Food Ontology Construction Mechanism for Diabetes Diet Care International Conference on Machine Learning and Cybernetics. Retrieved from https://ieeexplore.ieee.org/abstract/document/4370653

Joaquín Pérez-Ortega, (2019) Nelva Nely Almanza-Ortega, Andrea Vega-Villalobos, Rodolfo Pazos-Rangel, Crispín Zavala-Díaz and Alicia Martínez-Rebollar The Kmeans evolution April DOI : 10.5772/intechopen.85447 Retrieved from https://doi.org/10.5772/intechopen.85447

Maiyaporn Phanich; Phathrajarin Pholkul; Suphakant Phimoltares (2010). Food Recommendation System Using Clustering Analysis for Diabetic Patients, 
International Conference on Information Science and Applications. Retrieved from https://ieeexplore.ieee.org/abstract/document/5480416

Seo, Y., Kim, E. M., Choi, J. S., \& Park, C. Y. (2020). Using a Mobile-based Nutritional Intervention Application Improves Glycemic Control but Reduces the Intake of Some Nutrients in Patients with Gestational Diabetes Mellitus : A Case Series Study. Clinical Nutrition Research, 9(1), 73-79 Retrieved from https://doi.org/10.7762/cnr.2020.9.1.73 